\title{
Acetazolamide-Loaded Dynamic 7T MR Quantitative Susceptibility Mapping in Major Cerebral Artery Steno-Occlusive Disease: Comparison with PET
}

(D). Fujimoto, (DI. Uwano, (D). Sasaki, (D). Oshida, (DS. Tsutsui, (DW. Yanagihara, (D). Fujiwara, (D). Kobayashi, (D). Kubo, (D) Koshida, (D). Terasaki, and (D) K. Ogasawara

\begin{abstract}
BACKGROUND AND PURPOSE: Dynamic changes in cerebrovascular reactivity after acetazolamide administration vary markedly among patients with major cerebral arterial steno-occlusive disease. MR quantitative susceptibility mapping can dynamically quantify the cerebral magnetic susceptibility. The purpose of this study was to determine whether dynamic changes in susceptibility after administration of acetazolamide on 7T quantitative susceptibility mapping are associated with pre-existing states of CBV and the cerebral metabolic rate of oxygen in the cerebral hemispheres with major cerebral arterial steno-occlusive disease.
\end{abstract}

MATERIALS AND METHODS: Sixty-five patients underwent 7T MR imaging at baseline and at 5, 10, 15, and 20 minutes after acetazolamide administration. Differences between the susceptibility of venous structures and surrounding brain tissue were calculated in the quantitative susceptibility mapping images. Susceptibility differences at 5, 10, 15, and 20 minutes after acetazolamide administration relative to baseline were calculated in 97 cerebral hemispheres with major cerebral arterial steno-occlusive disease. CBV and the cerebral metabolic rate of oxygen were also calculated using ${ }^{15} \mathrm{O}$-gas PET in the resting state.

RESULTS: Dynamic changes of susceptibility after acetazolamide administration were classified into 3 patterns: abnormally increasing 5 or 10 minutes after acetazolamide administration; abnormally decreasing within 20 minutes after acetazolamide administration; and remaining unchanged after acetazolamide administration. CBV was significantly greater in the first pattern than in the latter 2 . The cerebral metabolic rate of oxygen differed significantly in descending order from the first to middle to last pattern.

CONCLUSIONS: Dynamic changes of susceptibility after acetazolamide administration on 7T MR quantitative susceptibility mapping are associated with pre-existing states of $\mathrm{CBV}$ and the cerebral metabolic rate of oxygen in major cerebral arterial steno-occlusive disease.

ABBREVIATIONS: $\mathrm{ACZ}=$ acetazolamide; $\mathrm{BOLD}=$ blood oxygen level-dependent; $\mathrm{CMRO}_{2}=$ cerebral metabolic rate of oxygen; $\mathrm{CVR}=$ cerebrovascular reactivity; $\mathrm{OEF}=$ oxygen extraction fraction; $\mathrm{QSM}=$ quantitative susceptibility mapping; $\mathrm{RS}=$ relative susceptibility

W hen the chronic progression of major cerebral arterial steno-occlusive disease reduces cerebral perfusion pressure, cerebrovascular autoregulatory mechanisms attempt to preserve CBF through the dilation of precapillary resistance vessels;

Received March 4, 2019; accepted after revision March 1, 2020.

From the Department of Neurosurgery (K.F., S.O., S.T., W.Y., S.F., M.K., Y.K., K.Y., K.O.), Division of Ultrahigh Field MRI, Institute for Biomedical Sciences (I.U., M.S.), and Cyclotron Research Center (K.T.), Iwate Medical University School of Medicine, Morioka, Japan.

This study was funded by a grant-in-aid for the Private University Research Branding Project from the Ministry of Education, Culture, Sports, Science and Technology-supported program; and scientific research from the Japan Society for the Promotion of Science (JP18K09002).

Please address correspondence to Kuniaki Ogasawara, M.D., Department of Neurosurgery, Iwate Medical University, Uchimaru 19-1, Morioka 020-8505, Japan; e-mail: kuogasa@iwate-med.ac.jp

- Indicates open access to non-subscribers at www.ajnr.org

Indicates article with supplemental on-line table.

http://dx.doi.org/10.3174/ajnr.A6508 this is referred to as stage 1 ischemia. ${ }^{1-3}$ In stage 1 ischemia, cerebrovascular reactivity (CVR) to acetazolamide (ACZ), which reflects the degree of cerebrovascular autoregulatory vasodilation, starts to decrease. ${ }^{1-3}$ However, autoregulatory capacity cannot compensate for further reductions in cerebral perfusion pressure, which result in a decline in CBF; this is referred to as stage 2 ischemia, or misery perfusion. ${ }^{1-3}$ CVR to ACZ is exhausted in this stage. ${ }^{1-3}$ In the clinical setting, CVR to ACZ is measured using perfusion SPECT, ${ }^{4}$ dynamic susceptibility contrast perfusion MR imaging, ${ }^{5}$ and transcranial Doppler ultrasonography. ${ }^{6}$

MR imaging is capable of quantifying the blood oxygen leveldependent (BOLD) changes in venous structures and/or brain parenchyma induced by differences in magnetic susceptibility between oxy- and deoxyhemoglobin. ${ }^{7-9} \mathrm{Wu}$ et al ${ }^{10}$ continuously measured CVR to ACZ using BOLD imaging with 3T MR imaging in patients with steno-occlusive disease of major cerebral arteries and demonstrated that overall, BOLD signal began 
increasing immediately after ACZ administration, approaching a plateau at $\sim 8.5$ minutes after administration. However, in some patients, BOLD signal showed a more severely reduced relative reactivity at 6-7 minutes after the initiation of $A C Z$ infusion and progressive partial recovery toward a pre-ACZ administration value at $10-11$ minutes. ${ }^{10}$ These findings suggested that dynamic changes to susceptibility after ACZ administration vary markedly among such patients. CVR to ACZ itself may depend on the preexisting state of vasodilation, which can be assessed using CBV, and pre-existing dilated vessels show little or no response to ACZ. ${ }^{1-3}$ We hypothesized that a pre-existing state of cerebral metabolism is another condition impacting CVR to ACZ; ACZ acts via enzyme reaction, and a reduction in this enzyme reaction causes a slow, decreased CVR to ACZ. Cerebral metabolism, which can be assessed using the cerebral metabolic rate of oxygen $\left(\mathrm{CMRO}_{2}\right)$, may be reduced in such a state.

A postprocessing technique known as quantitative susceptibility mapping (QSM) on MR imaging can quantify the magnetic susceptibility of venous structures and/or brain parenchyma from $\mathrm{T}^{*}$ weighted magnitude/phase images, which are easily obtained using a commercial scanner. ${ }^{11}$ Several investigators have measured CVR to ACZ using QSM on 3T MR imaging and suggested that QSM is sensitive to dynamic modulation of the oxygen extraction fraction (OEF) during hemodynamic augmentation. ${ }^{12}$ A recent study using QSM with a 7T scanner reported that it offered profound susceptibility effects and optimized postprocessing techniques. ${ }^{13}$ The scan time for this mapping is relatively short ( 3 minutes 25 seconds), and it may also enable imaging of dynamic changes in blood oxygenation levels in venous structures and/or brain parenchyma.

Therefore, the purpose of the present study was to determine whether dynamic changes in susceptibility after ACZ administration on 7T QSM are associated with pre-existing $\mathrm{CBV}$ and $\mathrm{CMRO}_{2}$ on PET in patients with ICA or MCA steno-occlusive disease.

\section{MATERIALS AND METHODS}

All procedures performed in studies involving human participants were in accordance with the institutional ethics committee, and written, informed consent was obtained from all subjects or their next of kin before patient participation.

\section{Healthy Subjects}

To obtain healthy control values from brain ${ }^{15} \mathrm{O}$-gas PET studies, we enrolled 10 healthy adult men (mean age, $47 \pm 8$ years; age range, 35-60 years) who had no history of diabetes mellitus, dyslipidemia, or hypertension and had an absence of asymptomatic lacunar infarction and leukoaraiosis on conventional brain MR imaging.

\section{Patient Inclusion Criteria}

We prospectively selected patients who provided written informed consent to participate and who met the following basic inclusion criteria: presence of clinical symptoms suggesting ischemic episodes in the MCA or ICA territory at 1-6 months before visiting our institution; useful residual function $(0,1$, or 2 on the mRS); presence of uni- or bilateral MCA (M1 portion) or extra- or intracranial ICA stenosis ( $>70 \%$ or $50 \%$ for the ICA and MCA, respectively) or occlusion on angiography; and absence of infarcts in the entire cortical area supplied by the M4 branch of the MCA on T1WI and T2WI, and DWI.

\section{MR Imaging Protocol and Generation of QSM}

A 7T MR scanner (Discovery MR950; GE Healthcare) with quadrature transmission and 32-channel receive head coils was used. QSM source data were obtained using a 3D spoiled gradient recalled acquisition technique with flow compensation (TR, $30 \mathrm{~ms}$; TE, $15 \mathrm{~ms}$; flip angle, $20^{\circ}$; FOV, $256 \mathrm{~mm}$; acquisition matrix size, $512 \times 256$; section thickness, $2 \mathrm{~mm}$; number of slices, 160 ; reconstruction voxel size after zero-fill interpolation, $0.5 \mathrm{~mm}^{3}$; and scan time, 3 minutes 25 seconds). ${ }^{13}$ Magnitude and real/imaginary phase images were regenerated from this acquisition.

First, each patient underwent the above-mentioned MR imaging at baseline. Next, ACZ (1000 mg; range, $13-19 \mathrm{mg} / \mathrm{kg}$ body weight) was dissolved in physiologic saline $(20 \mathrm{~mL})$; this solution was then administered intravenously for 1 minute. The MR imaging was performed in the same fashion 4 times so that each midscan time was 5, 10, 15, and 20 minutes after the end of intravenous administration of ACZ.

Before the start of this ACZ challenge study, 30 other cerebral hemispheres with ICA or MCA steno-occlusive disease underwent $5 \mathrm{MR}$ imaging scans with the same timing without ACZ administration to assess the minute-to-minute test-retest variability of susceptibility as measured using QSM.

QSM images were generated from the source images using an in-house program with a multiple dipole-inversion combination with $k$-space segmentation ${ }^{14}$ and regularization-enabled sophisticated harmonic artifact reduction for phase data methods, ${ }^{15}$ as described previously. ${ }^{13}$ A $2 \mathrm{D}$ Gaussian low-pass filter with a kernel size of $60 \%$ of the total image power in each section was applied to extract iron deposition in deep nuclei, hemosiderin deposition, dural sinuses, and large venous structures, and a $2 \mathrm{D}$ Gaussian high-pass filter of $2 \%$ was applied to extract small venous structures. ${ }^{13}$ Subsequently, small venous structures were determined by multiplying the Gaussian high-pass filter-processed binary images and the logical negations of Gaussian lowpass filter-processed binary images under the threshold for binarization of $\geq 2$ SDs. ${ }^{13}$

The difference between the average susceptibility of venous structures and surrounding brain tissue was calculated in each voxel of interest of $25 \mathrm{~mm}^{3}$ in the processed QSM images. ${ }^{13}$ The susceptibility difference was finally displayed with a smoothing procedure. To validate a correlation between baseline OEF obtained from QSM images and pre-existing OEF obtained on PET, we generated OEF maps from baseline QSM images before ACZ challenge in accordance with a previous study. ${ }^{13}$

\section{Brain ${ }^{15}$ O-Gas PET Study}

Patients who were scheduled to undergo MR imaging studies with the ACZ challenge underwent PET studies in a resting state without ACZ challenge. PET studies were performed using a SET-3000GCT/M scanner (PET/CT; Shimadzu) (full width at half maximum for in-plane and axial spatial resolutions of 3.5 and $4.2 \mathrm{~mm}$, respectively) within 5 days before MR imaging. Before the PET scans, a 3-minute transmission scan with a 


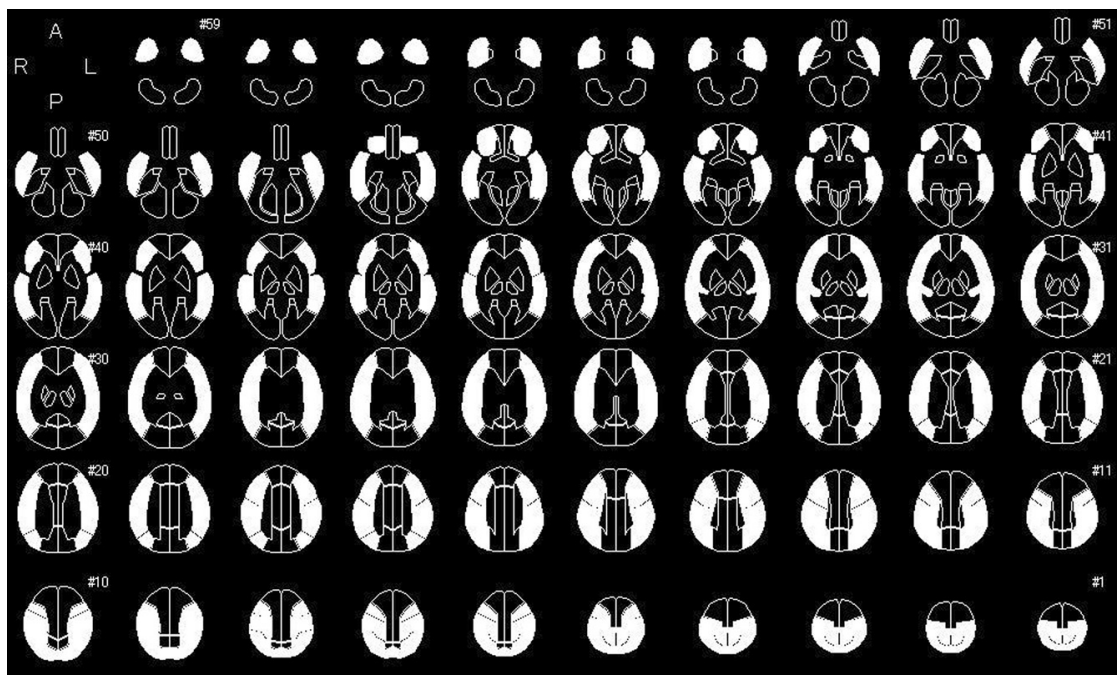

FIG 1. Diagrams showing the $318 \mathrm{ROIs}$ for the $3 \mathrm{D}$ stereotaxic ROI template. White ROls indicate territories perfused by bilateral MCAs.

cesium $137\left({ }^{137} \mathrm{Cs}\right)$ point source was conducted using a bismuth germanate transmission detector ring that was coaxially attached to the gadolinium silica oxide emission detector ring. Next, $\mathrm{CBF}$ was measured while the patient inhaled $\mathrm{C}^{15} \mathrm{O}_{2}$ continuously through a mask. The $\mathrm{CMRO}_{2}$ and OEF were measured during continuous inhalation of ${ }^{15} \mathrm{O}_{2}$; these data were collected for 5 minutes. $\mathrm{CBV}$ was measured during a single breath of $\mathrm{C}^{15} \mathrm{O}$. Finally, $\mathrm{CBF}, \mathrm{OEF}$, and $\mathrm{CMRO}_{2}$ were calculated using the steadystate method ${ }^{16}$ and then corrected on the basis of CBV. ${ }^{17}$

\section{Image Data Analyses}

Image data analyses were performed according to previously reported methods. ${ }^{13}$ By means of SPM, Version 12 (http://www. fil.ion.ucl.ac.uk/spm/software/spm12), ${ }^{18}$ PET images that were coregistered to QSM source images, as well as QSM images after Gaussian smoothing ( $\sigma=10$ pixel), were warped to Montreal Neurological Institute coordinates. Next, 318 constant ROIs were placed in both the cerebral and cerebellar hemispheres automatically using a 3D stereotaxic ROI template with SPM. ${ }^{19}$ The ROIs were then grouped into a total of 10 segments-callosomarginal, pericallosal, precentral, central, parietal, angular, temporal, posterior, hippocampal, and cerebellar-in each hemisphere according to the arterial supply. Five of these 10 segments (precentral, central, parietal, angular, and temporal) perfused by the MCA were then combined and defined as an MCA ROI (Fig 1).

Mean susceptibility differences on QSM images and CBF, $\mathrm{CBV}$, OEF, and $\mathrm{CMRO}_{2}$ on PET images were measured using image-analysis software (ITK-SNAP; www.itksnap.org) ${ }^{20}$ in the MCA ROI in the cerebral hemisphere with ICA or MCA stenoocclusive disease. In each MCA ROI of each cerebral hemisphere with ACZ challenge, relative susceptibility (RS, \%) was calculated as follows: $100 \times$ susceptibility difference at each time $(5,10,15$, or 20 minutes) after ACZ administration on QSM images/susceptibility difference in the baseline on QSM images. In each MCA ROI of each cerebral hemisphere without ACZ challenge, RS was also calculated as follows: $100 \times$ susceptibility difference on QSM images in the second, third, fourth, or fifth MR imaging/susceptibility difference on QSM images in the first MR imaging. When RS at $5\left(\mathrm{RS}_{5}\right), 10\left(\mathrm{RS}_{10}\right), 15$ $\left(\mathrm{RS}_{15}\right)$, or $20\left(\mathrm{RS}_{20}\right)$ minutes after ACZ administration was higher than the upper limit of the $95 \%$ CI of RS in the second, third, fourth, or fifth MR imaging, respectively, in the 30 cerebral hemispheres without ACZ challenge, the former RS was defined as abnormally increased. When $\mathrm{RS}_{5}, \mathrm{RS}_{10}, \mathrm{RS}_{15}$, or $\mathrm{RS}_{20}$ was lower than the lower limit of the $95 \%$ CI of RS in the second, third, fourth, or fifth MR imaging, respectively, in the 30 cerebral hemispheres without ACZ challenge, the former RS was defined as abnormally decreased. In each MCA ROI of each patient, a chronological change in RS after ACZ administration was classified on the basis of the presence or absence of an abnormal increase or decrease in RS at any time point.

In each MCA ROI of each patient, when CBV was greater than the upper limit of the $95 \%$ CI of control values obtained from 20 cerebral hemispheres of 10 healthy subjects, this ROI was defined as having abnormally elevated $\mathrm{CBV}$; when the $\mathrm{CMRO}_{2}$ was lower than the lower limit of the $95 \%$ CI of control values, this ROI was defined as showing abnormally reduced $\mathrm{CMRO}_{2}$.

\section{Statistical Analysis}

Data are expressed as the mean \pm SD. Pearson correlation coefficients were used to assess correlations between baseline QSM$\mathrm{OEF}$ and pre-existing PET-OEF. A difference in RS at each time point after ACZ administration was evaluated using the Friedman test. When this difference was significant, differences among RS values at each time after ACZ administration were compared using the Wilcoxon signed rank test followed by the Holm method. A difference in CBF, CBV, OEF, or $\mathrm{CMRO}_{2}$ among $\geq 3$ groups was evaluated using the Kruskal-Wallis test. When this difference was significant, differences among CBF, $\mathrm{CBV}$, OEF, or $\mathrm{CMRO}_{2}$ in each group were compared using the Mann-Whitney $U$ test followed by the Holm method. A difference in $\mathrm{CBV}$ or $\mathrm{CMRO}_{2}$ among 2 groups was evaluated using the Mann-Whitney $U$ test. $P$ values $<.05$ were considered statistically significant for all assessments.

\section{RESULTS}

During the 30-month study period, a total of 89 patients successfully underwent $7 \mathrm{~T} \mathrm{MR}$ imaging without severe adverse effects. In 4 of these patients, data sufficient to generate QSM were not obtained because of motion artifacts; these 4 patients were excluded from the analysis. Therefore, 85 patients (65 and 20 with and without ACZ challenge, respectively) were finally enrolled in the study.

AJNR Am J Neuroradiol 41:785-91 May 2020 www.ajnr.org 
Among these 85 patients (46 men, 39 women; mean age, $55 \pm$ 12 years; age range, 30-76 years), 60 had hypertension, 32 had diabetes mellitus, and 55 had dyslipidemia. Thirty-seven, 27, 3, and 17 patients had bilateral ICA (bilateral extracranial diseases in 27; bilateral intracranial diseases in 5; unilateral extracranial disease and contralateral intracranial disease in 5), unilateral ICA (extracranial disease in 22; intracranial disease in 5), the $\mathrm{M} 1$ portion diseases of bilateral MCA, and unilateral M1 portion steno-occlusive disease, respectively. The remaining patient had unilateral extracranial ICA and contralateral M1 portion steno-occlusive diseases. Therefore, 127 MCA ROIs of the 127 cerebral hemispheres with MCA or ICA steno-occlusive disease (97 and 30 with and without ACZ challenge, respectively) were eventually analyzed.

The 95\% CI of RS values in the second, third, fourth, and fifth MR imaging in 30 MCA ROIs without ACZ challenge were 91.85\%-108.55\%, 90.81\%-109.33\%, 90.59\%-109.49\%, and 90.70\%$109.22 \%$, respectively. No difference was observed among each RS. The 95\% CIs of CBV and $\mathrm{CMRO}_{2}$ obtained from 20 MCA ROIs of 10 healthy subjects were $2.45-5.01 \mathrm{~mL} / 100 \mathrm{~g}$ and $2.31-4.70 \mathrm{~mL} / 100$ $\mathrm{g} / \mathrm{min}$, respectively.

A significant correlation was observed between baseline QSM-OEF and pre-existing PET-OEF $(r=0.865 ; P \leq .0001)$ (Fig 2).

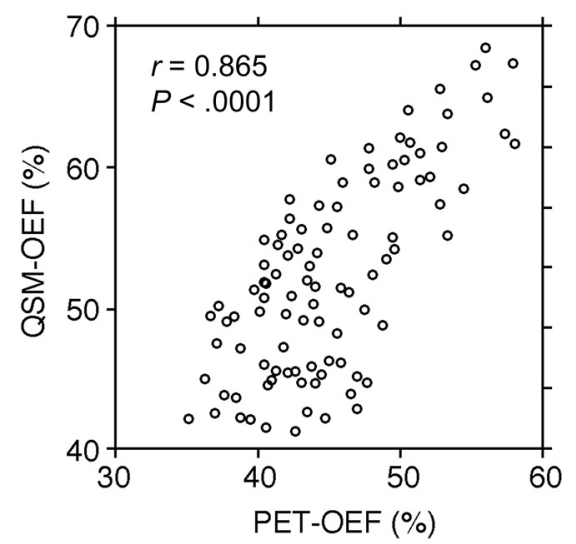

FIG 2. Correlations between baseline QSM-OEF before ACZ administration and pre-existing PET-OEF in 97 MCA ROIs.
Figure 3 shows chronological changes in RS after ACZ administration for the 97 MCA ROIs. Patterns of these changes were classified into the following 3 groups: RS was abnormally increased at $\geq 1$ time point in 22 MCA ROIs; RS was abnormally decreased at $\geq 1$ time point in 47 MCA ROIs; and RS remained within the $95 \%$ CI of controls at all time points (defined as unchanged RS) in 28 MCA ROIs. None of the MCA ROIs exhibited an abnormally increased RS and an abnormally decreased RS at different time points.

Of the 22 MCA ROIs with abnormally increased RS, 2 exhibited abnormally increased RS at all time points; 1 exhibited abnormally increased $\mathrm{RS}_{5}, \mathrm{RS}_{10}$, and $\mathrm{RS}_{15}$; eleven exhibited abnormally increased $\mathrm{RS}_{5}$ and $\mathrm{RS}_{10}$; one exhibited abnormally increased $\mathrm{RS}_{5}$ and $\mathrm{RS}_{20}$; five exhibited abnormally increased $\mathrm{RS}_{5}$ alone; and 2 exhibited abnormally increased $\mathrm{RS}_{10}$ alone.

Of the 47 MCA ROIs with abnormally decreased RS, 18 exhibited abnormally decreased RS at all time points; 3 exhibited abnormally decreased $\mathrm{RS}_{5}, \mathrm{RS}_{10}$, and $\mathrm{RS}_{15}$; three exhibited abnormally decreased $\mathrm{RS}_{5}, \mathrm{RS}_{10}$, and $\mathrm{RS}_{20}$; two exhibited abnormally decreased $\mathrm{RS}_{5}, \mathrm{RS}_{15}$, and $\mathrm{RS}_{20}$; one exhibited abnormally decreased $\mathrm{RS}_{10}$, $\mathrm{RS}_{15}$, and $\mathrm{RS}_{20}$; one exhibited abnormally decreased $\mathrm{RS}_{5}$ and $\mathrm{RS}_{10}$; one exhibited abnormally decreased $\mathrm{RS}_{15}$ and $\mathrm{RS}_{20}$; nine exhibited abnormally decreased $\mathrm{RS}_{5}$ alone; 3 exhibited abnormally decreased $\mathrm{RS}_{10}$ alone; 2 exhibited abnormally decreased $\mathrm{RS}_{15}$ alone; and 4 exhibited abnormally decreased $\mathrm{RS}_{20}$ alone.

In the 22 MCA ROIs with abnormally increased RS, RS differed significantly among 4 time points: $\mathrm{RS}_{5}(113.84 \pm 6.28 \%$; $P=.0081$ for $\mathrm{RS}_{10} ; P<.0001$ for $\mathrm{RS}_{15} ; P<.0001$ for $\left.\mathrm{RS}_{20}\right), \mathrm{RS}_{10}$ $\left(111.58 \pm 5.06 \% ; P=.0001\right.$ for $\mathrm{RS}_{15} ; P=.0001$ for $\left.\mathrm{RS}_{20}\right), \mathrm{RS}_{15}$ $\left(104.72 \pm 5.43 \% ; P=.0019\right.$ for $\left.\mathrm{RS}_{20}\right)$, and $\mathrm{RS}_{20}(100.93 \pm 5.55 \%)$ in descending order (Fig 3). In the 47 MCA ROIs with abnormally decreased RS, no significant differences were observed among $\mathrm{RS}_{5}(88.69 \pm 5.56 \%), \mathrm{RS}_{10}(88.55 \pm 5.98 \%), \mathrm{RS}_{15}(88.71$ $\pm 5.87 \%)$, and $\mathrm{RS}_{20}(89.00 \pm 5.83 \%)$. In the $28 \mathrm{MCA}$ ROIs with unchanged RS, no significant differences were observed among $\mathrm{RS}_{5}(97.89 \pm 4.89 \%), \mathrm{RS}_{10}(99.06 \pm 4.47 \%), \mathrm{RS}_{15}$ (97.85 \pm $3.74 \%)$, and $\mathrm{RS}_{20}(97.80 \pm 3.78 \%)$.

The On-line Table shows comparisons of pre-existing CBV and $\mathrm{CMRO}_{2}$ among MCA ROIs with abnormally increased RS, abnormally decreased RS, unchanged RS, and MCA ROIs in healthy subjects as controls. CBV was significantly greater in
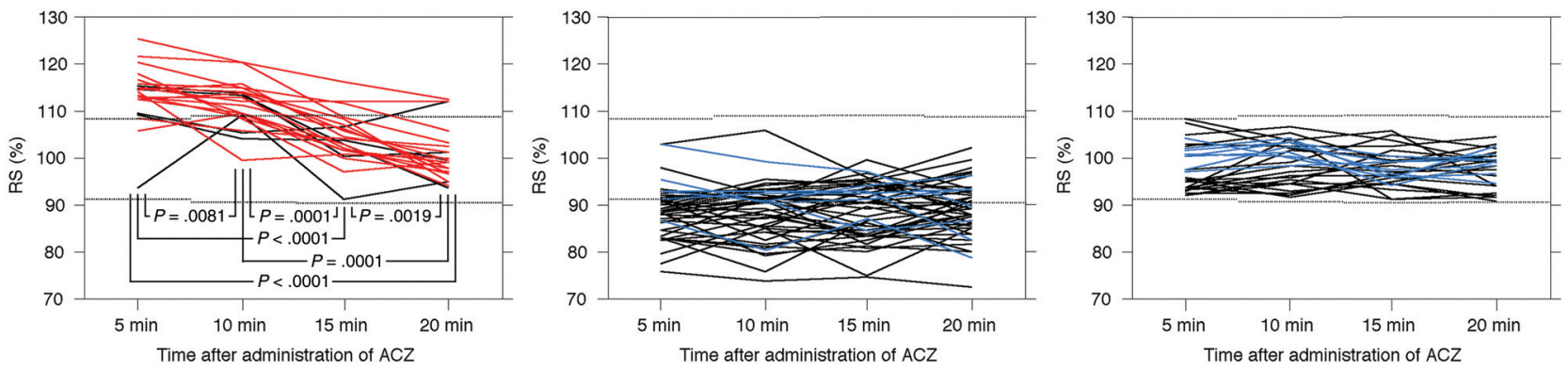

FIG 3. Chronological changes in RS after ACZ administration in $97 \mathrm{MCA}$ ROIs. Left, middle, and right graphs show the $22 \mathrm{MCA}$ ROIs with abnormally increased RS at $\geq 1$ time point, the 47 MCA ROls with abnormally decreased RS at $\geq 1$ time point, and the 28 MCA ROls without abnormally increased or decreased RS at any time point, respectively. Red, blue, and black lines denote ROls with abnormally elevated CBV, an abnormally decreased cerebral metabolic rate of oxygen, and neither, respectively. Upper and lower dotted horizontal lines denote the upper and lower limits of the $95 \% \mathrm{Cl}$ of controls without ACZ challenge, respectively. 
MCA ROIs with abnormally increased RS than in those with abnormally decreased RS, unchanged RS, or controls. No significant differences in CBV were identified in other any comparisons. Of the 22 MCA ROIs with abnormally increased RS, 17 (77\%) had abnormally elevated CBV. In contrast, none of the patients with abnormally decreased RS or unchanged RS had abnormally elevated $\mathrm{CBV}$. No significant differences in $\mathrm{CMRO}_{2}$ were apparent between controls and MCA ROIs with abnormally increased RS. Regarding other comparisons, the $\mathrm{CMRO}_{2}$ differed significantly in descending order, as follows: controls or abnormally increased RS, abnormally decreased RS, and unchanged RS. Of the 28 MCA ROIs with unchanged RS, 8 (29\%) showed abnormally reduced $\mathrm{CMRO}_{2}$. In contrast, of the $47 \mathrm{MCA}$ ROIs with abnormally decreased RS, only 7 (14\%) had abnormally reduced $\mathrm{CMRO}_{2}$, and no MCA ROIs with abnormally increased RS had abnormally reduced $\mathrm{CMRO}_{2}$. Furthermore, in the 47 MCA ROIs with abnormally decreased RS, whereas CBV did not differ significantly in the 11 MCA ROIs with unchanged $\mathrm{RS}_{5}(3.35 \pm 0.84 \mathrm{~mL} / 100 \mathrm{~g})$ and the 36 MCA ROIs with abnormally decreased $\mathrm{RS}_{5}(3.40 \pm 0.3862 \mathrm{~mL} / 100 \mathrm{~g}), \mathrm{CMRO}_{2}$ was significantly lower in the former MCA ROIs $(2.65 \pm 0.52 \mathrm{~mL} / 100$ $\mathrm{g} / \mathrm{min})$ than in the latter $(3.06 \pm 0.38 \mathrm{~mL} / 100 \mathrm{~g} / \mathrm{min} ; P=.0182)$.

Representative chronological images of susceptibility difference obtained from QSM after ACZ administration and PET images performed before MR imaging in a patient with abnormally increased CBV in the symptomatic cerebral hemisphere are shown in Fig 4.

\section{DISCUSSION}

The present study demonstrated that dynamic changes in susceptibility after ACZ administration on 7T QSM are associated with pre-existing $\mathrm{CBV}$ and $\mathrm{CMRO}_{2}$ on PET in patients with ICA or MCA steno-occlusive disease.

In this study, ACZ was administered intravenously for 1 minute. This method has been applied for measuring CVR on brain perfusion SPECT in adult patients with ICA or MCA steno-occlusive disease. ${ }^{4,21}$ Considering the adverse effects of ACZ administration performed in the same fashion, $63 \%$ of patients reportedly developed only minor symptoms such as headache, nausea, dizziness, tinnitus, numbness of the extremities, and general malaise, and these symptoms resolved within 72 hours after ACZ administration. ${ }^{22}$ These patients experienced neither severe general symptoms nor neurologic deficits. $^{22}$ Similarly, no patients in the present study experienced such severe adverse effects due to ACZ administration.

A study using ${ }^{15} \mathrm{O}$ PET demonstrated that while CBF increased at 10 and 20 minutes after $\mathrm{ACZ}$ administration in healthy humans, $\mathrm{CMRO}_{2}$ was unchanged compared with preadministration. ${ }^{23}$ Changes in susceptibility after ACZ administration on QSM theoretically reflect both changes in $\mathrm{CBF}$ and oxygen metabolism by ACZ. Although whether $\mathrm{CMRO}_{2}$ after ACZ administration is constant in patients with cerebrovascular diseases remains unknown, dynamic changes in susceptibility after ACZ administration on QSM may more strongly reflect changes in CBF than changes in oxygen metabolism: Increases and decreases in the susceptibility may predominantly indicate decreases and increases in $\mathrm{CBF}$, respectively. One study of CVR to ACZ using QSM on 3T MR imaging showed that $\mathrm{OEF}$ decreased from baseline to 16 minutes

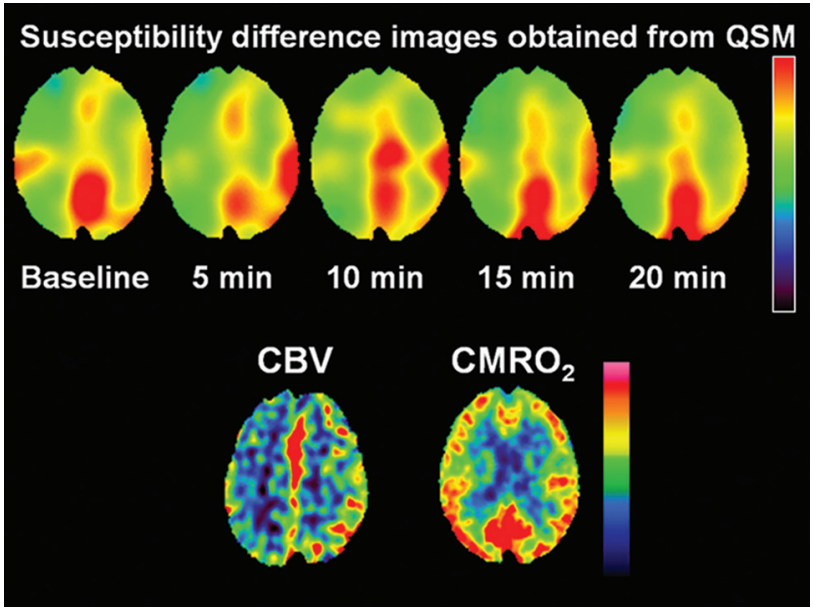

FIG 4. A 55-year-old man with transient ischemic attacks of right hemiparesis and bilateral MCA stenosis. Susceptibility on susceptibility difference images (upper) obtained from QSM is increased at 5 and 10 minutes after ACZ administration in the left cerebral hemisphere compared with baseline, suggesting early steal phenomenon. The susceptibility in that hemisphere begins to decline at 15 minutes after ACZ administration and reaches below baseline at 20 minutes after ACZ administration. By contrast, susceptibility in the right cerebral hemisphere is decreased at 5 minutes after ACZ administration compared with baseline and is gradually increased at 10 and 15 minutes after ACZ administration. However, the susceptibility in that hemisphere at 20 minutes after $\mathrm{ACZ}$ administration remains below baseline. ${ }^{15} \mathrm{O}$-gas PET images at resting state (lower images) show an increase in $\mathrm{CBV}$ and $\mathrm{CMRO}_{2}$ within normal limits in the left cerebral hemisphere.

after ACZ administration in the cerebral hemisphere with anterior circulation steno-occlusive disease, as well as in the cerebral hemisphere without such disease, and the decrease was more substantial in the latter. ${ }^{12}$ These changes in susceptibility might be caused by changes of CBF. ${ }^{12}$

In the present study, dynamic changes of susceptibility after ACZ administration were classified into 3 patterns: abnormally increasing, peaking at 5 or 10 minutes after ACZ administration and later returning toward baseline; abnormally decreasing within 20 minutes after ACZ administration and maintaining this decrease; and remaining unchanged after ACZ administration. A dynamic change comprising an abnormal increase in susceptibility early after ACZ administration and subsequent return was comparable with a finding obtained using continuous measurements of BOLD signal after ACZ administration. ${ }^{10}$ In MCA ROIs with such dynamic changes, pre-existing $\mathrm{CBV}$ was abnormally elevated and pre-existing $\mathrm{CMRO}_{2}$ was normally maintained. Mean transit time (MMT) is a function of CBV/CBF, and an increase in $\mathrm{CBV}$ usually implies a long MMT in MCA or ICA steno-occlusive disease. A long MMT can result in low drug delivery, leading to little or no CVR immediately after ACZ administration in the region affected by such a condition, whereas CVR in surrounding or other brain tissues with a normal MMT reacts rapidly because of normal drug delivery. In addition, the small arterioles or intraparenchymal vessels are dilated in regions with an increase in CBV. These pre-existing dilated 
vessels cannot respond immediately after ACZ administration, whereas normal vessels in surrounding or other brain tissue rapidly dilate. These differences in CVR to ACZ administration lead to a sudden reduction in cerebral perfusion pressure of normal vessels relative to pre-existing dilated vessels. This dynamically developed pressure gradient may result in the early steal of blood flow from pre-existing dilated vessels to normal vessels. Subsequently, pre-existing dilated vessels may gradually respond to $\mathrm{ACZ}$, and a vasodilation effect of ACZ in normal vessels may gradually reduce with time. Such mechanisms could explain the abnormal increase in susceptibility early after ACZ administration (early steal phenomenon) and subsequent return.

A dynamic change of the abnormal decrease in susceptibility within 20 minutes after ACZ administration and maintenance of this decrease might imply an increase in $\mathrm{CBF}$ - that is, normal CVR to ACZ. Actually, in MCA ROIs showing such a dynamic change, pre-existing $\mathrm{CBV}$ was normal. Pre-existing $\mathrm{CMRO}_{2}$ was reduced compared with that in controls but remained within normal limits in $86 \%$ of MCA ROIs showing that susceptibility change. On the other hand, pre-existing $\mathrm{CMRO}_{2}$ was reduced more in MCA ROIs with an abnormal decrease in susceptibility $\geq 10$ minutes after ACZ administration than in those with an abnormal decrease in susceptibility 5 minutes after ACZ administration. Because CBV did not differ significantly between these 2 groups, the difference in dynamic change after ACZ administration might be attributable to pre-existing $\mathrm{CMRO}_{2}$. ACZ acts via carbonic anhydrase, and a reduction in its activity slows CVR to ACZ. The activity of carbonic anhydrase may depend on cerebral metabolism. This hypothesis may also explain the maintenance of unchanged susceptibility after ACZ administration. In MCA ROIs with such a dynamic change in susceptibility, pre-existing $\mathrm{CBV}$ was normal, but pre-existing $\mathrm{CMRO}_{2}$ was quite markedly reduced; this finding suggests that the activity of carbonic anhydrase was severely reduced and cerebral vessels were unresponsive to ACZ.

Using continuous measurement of CVR to ACZ with BOLD imaging, $\mathrm{Wu}$ et $\mathrm{al}^{10}$ demonstrated that BOLD signal began increasing gradually following ACZ administration, approaching a plateau at $\sim 8.5$ minutes after administration in the cerebral hemisphere without ICA or MCA steno-occlusive disease. This dynamic change in susceptibility differed compared with that of MCA ROIs with normal CBV and normal $\mathrm{CMRO}_{2}$ in the present study: an abnormal decrease in susceptibility 5 minutes after ACZ administration and maintenance of this decrease. The difference in peak susceptibility may be due to the method of ACZ administration. ACZ (1000 mg) was administered intravenously for 35 minutes in the study by Wu et al and for 1 minute in the present study.

A preoperative decrease in CVR to $\mathrm{ACZ}$ or a preoperative increase in $\mathrm{CBV}$ in the cerebral hemisphere ipsilateral to carotid endarterectomy or carotid artery stent placement for cervical ICA stenosis or arterial bypass surgery for Moyamoya disease has been identified as a powerful and independent predictor of postoperative cerebral hyperperfusion. ${ }^{21,24,25}$ Postoperative cerebral hyperperfusion induces unilateral headache, face and eye pain, seizure, and focal symptoms that occur secondary to cerebral edema or intracerebral hemorrhage. ${ }^{21,25}$ This phenomenon, even when asymptomatic, induces slight-but-diffuse brain damage, resulting in postoperative cognitive decline. ${ }^{26}$ Identification of reduced CVR to ACZ or elevated CBV therefore enables improved risk stratification for such patients. However, the predictive accuracy of CVR to ACZ on brain perfusion SPECT is not always high, with a positivepredictive value of $<50 \%$. ${ }^{21,24}$ Our findings that dynamic changes in susceptibility, particularly susceptibility 5 minutes after ACZ administration, detected increased CBV with high positive (77\%) and negative (100\%) predictive values suggests that these dynamic changes may predict the risk of cerebrovascular adverse events more accurately than conventional measurements of CVR to ACZ. In addition, the examination time of the present method using $7 \mathrm{~T}$ QSM was $<12$ minutes if the susceptibility change was measured until a midscan time of 5 minutes after ACZ administration.

QSM source data were obtained with a section thickness of $2 \mathrm{~mm}$. Such a thick section may reduce the accuracy of susceptibility on QSM due to the partial volume effect for small veins. ${ }^{11,13}$ As another limitation, the scan time for 7T QSM is 3 minutes $25 \mathrm{sec}-$ onds longer than that for BOLD or QSM imaging with 3T MR imaging. ${ }^{10}$ Shortening the scan time may help clarify dynamic changes in susceptibility after ACZ administration. Furthermore, considerable variation in cerebral hemodynamics might be anticipated within an MCA ROI, especially in the borderzone, ${ }^{12}$ and use of the MCA ROI might reduce such hemodynamic variation. Ranges of CBV in the cerebral gray matter are quite large. This high heterogeneity might also be reduced using MCA ROI analysis.

\section{CONCLUSIONS}

The present study demonstrated that the dynamic changes in susceptibility after ACZ administration on 7T QSM are associated with pre-existing $\mathrm{CBV}$ and $\mathrm{CMRO}_{2}$ on PET in patients with ICA or MCA steno-occlusive disease.

Disclosures: Makoto Sasaki—UNRELATED: Consultancy: Actelion Pharmaceuticals, Micron Technology; Grants/Grants Pending: Hitachi; Ministry of Education, Culture, Sports, Science and Technology; Agency for Medical Research and Development; Ministry of Health, Labour and Welfare*; Payment for Lectures Including Service on Speakers Bureaus: Actelion Pharmaceuticals, Mitsubishi Tanabe Pharma, Bayer AG, Nihon Medi-Physics Co., Ltd, Eisai Co., Ltd, FujiFilm, Daiichi Sankyo, Boehringeringelheim.jp, Otsuka America Pharmaceutical, CHUGAI PHARMACEUTICAL, Astellas Pharma, Ono Pharmaceutical. *Money paid to the institution.

\section{REFERENCES}

1. Baron JC, Bousser MG, Rey A, et al. Reversal of focal “misery-perfusion syndrome" by extra-intracranial arterial bypass in hemodynamic cerebral ischemia: a case study with ${ }^{15} \mathrm{O}$ positron emission tomography. Stroke 1981;12:454-59 CrossRef Medline

2. Gibbs JM, Leenders KL Wise RJ, et al. Evaluation of cerebral perfusion reserve in patients with carotid-artery occlusion. Lancet 1984;1:310-14 CrossRef

3. Powers WJ, Raichle ME. Positron emission tomography and its application to the study of cerebrovascular disease in man. Stroke 1985;16:361-76 CrossRef Medline

4. Ogasawara K, Ito $\mathrm{H}$, Sasoh M, et al. Quantitative measurement of regional cerebrovascular reactivity to acetazolamide using ${ }^{123} \mathrm{I}-\mathrm{N}$ isopropyl-p-iodoamphetamine autoradiography with SPECT: validation study using $\mathbf{H}_{\mathbf{2}}{ }^{\mathbf{1 5}} \mathbf{O}$ with PET. J Nucl Med 2003;44:520-25 Medline

5. Wiart M, Berthezène $\mathrm{Y}$, Adeleine $\mathrm{P}$, et al. Vasodilatory response of border zones to acetazolamide before and after endarterectomy: an echo planar imaging-dynamic susceptibility contrast-enhanced 
MRI study in patients with high-grade unilateral internal carotid artery stenosis. Stroke 2000;31:1561-65 CrossRef Medline

6. Aries M, Elting JW, De Keyser J, et al. Cerebral autoregulation in stroke: a review of transcranial Doppler studies. Stroke 2010;41:26972704 CrossRef Medline

7. Zhang J, Liu T, Gupta A, et al. Quantitative mapping of cerebral metabolic rate of oxygen (CMRO2) using quantitative susceptibility mapping (QSM). Magn Reson Med 2015;74:945-52 CrossRef Medline

8. De Vis JB, Petersen ET, Bhogal A, et al. Calibrated MRI to evaluate cerebral hemodynamics in patients with an internal carotid artery occlusion. J Cereb Blood Flow Metab 2015;35:1015-23 CrossRef Medline

9. Liu Z, Li Y. Cortical cerebral blood flow, oxygen extraction fraction, and metabolic rate in patients with middle cerebral artery stenosis or acute stroke. AJNR Am J Neuroradiol 2016;37:607-14 CrossRef Medline

10. Wu J, Dehkharghani S, Nahab F, et al. Acetazolamide-augmented dynamic BOLD (aczBOLD) imaging for assessing cerebrovascular reactivity in chronic steno-occlusive disease of the anterior circulation: an initial experience. Neuroimage Clin 2017;13:116-22 CrossRef Medline

11. Kudo K, Liu T, Murakami T, et al. Oxygen extraction fraction measurement using quantitative susceptibility mapping: comparison with positron emission tomography. J Cereb Blood Flow Metab 2016;36:1424-33 CrossRef Medline

12. Leatherday C, Dehkharghani S, Nahab F, et al. Cerebral MR oximetry during acetazolamide augmentation: beyond cerebrovascular reactivity in hemodynamic failure. J Magn Reson Imaging 2019;50:175-82 CrossRef Medline

13. Uwano I, Kudo K, Sato R, et al. Noninvasive assessment of oxygen extraction fraction in chronic ischemia using quantitative susceptibility mapping at 7 Tesla. Stroke 2017;48:2136-41 CrossRef Medline

14. Sato R, Shirai T, Taniguchi Y, et al. Quantitative susceptibility mapping using the multiple dipole-inversion combination with $\mathrm{k}$ space segmentation method. Magn Reson Med Sci 2017;16:340-50 CrossRef Medline

15. Sun H, Wilman AH. Background field removal using spherical mean value filtering and Tikhonov regularization. Magn Reson Med 2014;71:1151-57 CrossRef Medline

16. Frackowiak RS, Lenzi GL, Jones T, et al. Quantitative measurement of regional cerebral blood flow and oxygen metabolism in man using $15 \mathrm{O}$ and positron emission tomography: theory, procedure, and normal values. J Comput Assist Tomogr 1980;4:727-36 CrossRef Medline
17. Lammertsma AA, Jones T. Correction for the presence of intravascular oxygen-15 in the steady-state technique for measuring regional oxygen extraction ratio in the brain, 1; description of the method. J Cereb Blood Flow Metab 1983;3:416-24 CrossRef Medline

18. Ashburner J. SPM: a history. Neuroimage 2012;62:791-800 CrossRef Medline

19. Takeuchi R, Matsuda H, Yoshioka K, et al. Cerebral blood flow SPET in transient global amnesia with automated ROI analysis by 3DSRT. Eur J Nucl Med Mol Imaging 2004;31:578-89 CrossRef Medline

20. Yushkevich PA, Piven J, Hazlett HC, et al. User-guided 3D active contour segmentation of anatomical structures: significantly improved efficiency and reliability. Neuroimage 2006;31:1116-28 CrossRef Medline

21. Oshida S, Ogasawara K, Saura H, et al. Does preoperative measurement of cerebral blood flow with acetazolamide challenge in addition to preoperative measurement of cerebral blood flow at the resting state increase the predictive accuracy of development of cerebral hyperperfusion after carotid endarterectomy? Results from $\mathbf{5 0 0}$ cases with brain perfusion single-photon emission computed tomography study. Neurol Med Chir (Tokyo) 2015;55:141-48 CrossRef Medline

22. Saito H, Ogasawara K, Suzuki T, et al. Adverse effects of intravenous acetazolamide administration for evaluation of cerebrovascular reactivity using brain perfusion single-photon emission computed tomography in patients with major cerebral artery steno-occlusive diseases. Neurol Med Chir (Tokyo) 2011;51:479-83 CrossRef Medline

23. Okazawa $H$, Yamauchi $H$, Sugimoto K, et al. Effects of acetazolamide on cerebral blood flow, blood volume, and oxygen metabolism: a positron emission tomography study with healthy volunteers. $J$ Cereb Blood Flow Metab 2001;21:1472-79 CrossRef Medline

24. Yoshimura S, Kitajima H, Enomoto Y, et al. Staged angioplasty for carotid artery stenosis to prevent postoperative hyperperfusion. Neurosurgery 2009;64(3 Suppl):ons122-28; discussion ons128-29 CrossRef Medline

25. Uchino $\mathrm{H}$, Kuroda S, Hirata K, et al. Predictors and clinical features of postoperative hyperperfusion after surgical revascularization for Moyamoya disease: a serial single photon emission CT/positron emission tomography study. Stroke 2012;43:2610-16 CrossRef Medline

26. Chida K, Ogasawara K, Suga Y, et al. Postoperative cortical neural loss associated with cerebral hyperperfusion and cognitive impairment following carotid endarterectomy: ${ }^{123}$ I-iomazenil SPECT study. Stroke 2009;40:448-53 CrossRef Medline 